\title{
On Couple-Group Consensus of Multiagent Networks with Communication and Input Time Delays
}

\author{
Liang-hao Ji and Xin-yue Zhao \\ Chongqing Key Laboratory of Computational Intelligence, Chongqing University of Posts and Telecommunications, \\ Chongqing 400065, China
}

Correspondence should be addressed to Liang-hao Ji; lianghao.ji@gmail.com

Received 19 August 2016; Accepted 31 October 2016

Academic Editor: Guanghui Wen

Copyright @ 2016 L.-h. Ji and X.-y. Zhao. This is an open access article distributed under the Creative Commons Attribution License, which permits unrestricted use, distribution, and reproduction in any medium, provided the original work is properly cited.

\begin{abstract}
This paper investigated the couple-group consensus problems of the multiagent networks with the influence of communication and input time delays. Based on the frequency-domain theory, some algebraic criteria are addressed analytically. From the results, it is found that the input time delays and the coupling strengths between agents of the systems play a crucial role in reaching group consensus. The convergence of the system is independent of the communication delays, but it will affect the convergence rate of the system. Finally, several simulated examples are provided to verify the validity and correctness of our theoretical results.
\end{abstract}

\section{Introduction}

Over the past few years, increasing attention has been paid to the investigation of distributed cooperative control of multiagent networks due to its broad application in many areas, such as distributed sensor networks [1], congestion control in networks $[2,3]$, and flocking $[4,5]$. As a fundamental problem of cooperative control, consensus problem of multiagent networks has become the focus of researchers in many fields. Consensus of multiagent networks usually means a group of agents converging to a consistent state through sharing local communication with their neighbors. Recently, lots of works about consensus have been reported [6-11].

Group consensus is an extended consensus problem, which means that the agents in a network reach more than one consistent state. Namely, some agents in one subgroup can reach a consistent state while there is no consensus among different subgroups of the whole networks.

Recently, many reports about group consensus have been listed, such as, for second-order multiagent networks with time delays, the group consensus problem investigated in [12]. Based on the semitensor product of matrices and the vertex coloring of graphs, the group consensus problem of the multiagent network is discussed [13]. Yi et al. [14] discussed the group consensus issue of linearly coupled multiagent networks, where the relation between Laplacian and number of groups was derived. Based on some assumptions, Tan et al. [15] obtained some sufficient and necessary conditions to guarantee solvability of group consensus problems. $\mathrm{Hu}$ et al. [16] studied average-group consensus problems with undirected networks by designing a novel hybrid protocol. In [17], Zhao et al. discussed couple-group consensus problems of second-order multiagent networks with fixed and stochastic switching topologies and provided a sufficient and necessary condition for the mean-square couple-group consensus. In [18], conditions for reaching group consensus with centralized and decentralized event-triggered control were illustrated, respectively. Moreover, pinning control strategies have been introduced for reducing the cost of network control in [19-23]. In [24, 25], Yu and Wang addressed group consensus problems of networks with directed graphs and strongly connected and balanced topology. Furthermore, in [26, 27], $\mathrm{Yu}$ and Wang discussed the group consensus problem with communication delays and switching topologies. For the networks with strongly connected and balanced topology, Wang and Uchida [28] investigated the group consensus of multiagent networks with communication delays. Based on the networks with bipartite topology, group consensus of first-order multiagent networks with and without time delays were investigated in [29], respectively. Du et al. [30] 
further extended the conclusions in [29] and obtained an upper bound of time delay. Ji et al. [31] considered the group consensus of undirected and connected bipartite graphs, respectively, and proposed a sufficient condition for group consensus. In [32], Lianghao and Xinyue discussed the group consensus problem for second-order multiagent networks with the influence of input and communication time delays.

It is worth noting that the related research works mentioned above either only consider the influence of communication delays or simply discuss the special case that the communication delay is equal to the input delay, such as in [2427, 29-31]. Meanwhile, most of the works are based on the special topologies [16, 24, 25, 28-31], for instance, undirected, strongly connected, and balanced topologies. As we know, time delays objectively exist in the networks and are usually different. Meanwhile, time delays can degrade or even destroy the stable performance of the complex systems. Inspired by the related works, this paper focuses on discussing the couple-group consensus of multiagent networks under the influence of time delays. The main contributions are listed as follows: firstly, this paper investigated the influence of different time delays for the multiagent networks with the asymmetric topology. By using frequency-domain theory, the criteria which can guarantee the achievement of couplegroup consensus are proposed. Secondly, either the algebraic criteria we obtained are less conservative than the findings in related works or the relevant research works can be seen as the special cases of our works.

The rest of the paper is organized as follows. In Section 2, some relevant concepts about graph theory and model formulation are reviewed. In Section 3, the problems of group consensus for multiagent networks with multiple time delays are analyzed. Several numerical experiments are illustrated in Section 4. Finally, some conclusions and future work are drawn.

\section{Preliminaries}

Based on graph theory, an agent and the information interaction between them in a multiagent networks can be described by $G=(V, E, A)$, where $V=\left\{v_{1}, v_{2}, \ldots, v_{N}\right\}$ is node set, $\mathbb{N}=$ $\{1,2, \ldots, N\}$ denotes node index set, $E \subseteq V \times V$ is the edge set, and $A=\left\{a_{i j}\right\} \in \mathbb{R}^{N \times N}$ is the weighted adjacency matrix. The neighbor of node $v_{i}$ is denoted by $N_{i}=\left\{v_{j} \in V:\left(v_{i}, v_{j}\right) \in E\right\}$. For convenience, suppose $a_{i j}>0$ if $v_{j} \in N_{i}$ and $a_{i i}=0$ for $\forall i \in \mathbb{N}$. The Laplacian matrix of $G$ is denoted by $L=D-A$, where $D=\operatorname{diag}\left\{d_{i}, i \in \mathbb{N}\right\}$ and $d_{i}=\sum_{v_{j} \in N_{i}} a_{i j}$.

For first-order multiagent networks, the dynamics of the system can be listed as

$$
\dot{x}_{i}(t)=u_{i}(t),
$$

where $x_{i}(t), u_{i}(t) \in \mathbb{R}^{k}$ denotes the position state and control input of the agent $i$ at time $t$, respectively. For simplicity, we just only consider the case $x_{i}(t), u_{i}(t) \in \mathbb{R}$. When $k>1$, it is easy to promote the results by applying the Kronecker product.

Next, some related definitions and lemmas will be listed firstly.
Definition 1. For $\forall i, j \in \mathbb{N}$, the first-order multiagent networks (1) can be said to achieve consensus asymptotically if and only if $\lim _{t \rightarrow \infty}\left\|x_{i}(t)-x_{j}(t)\right\|=0$.

Definition 2 (see [33]). For a bipartite graph $g=(V, E)$ which has the following properties, where $V$ and $E$ denote its vertex and edge sets:

(i) $V_{1} \cup V_{2}=V$ and $V_{1} \cap V_{2}=\varnothing$, where $V_{1}$ and $V_{2}$ are the two vertex subsets;

(ii) $\forall e=(x, y) \in E$, where $x \in V_{1}$ and $y \in V_{2}$.

Assumes the topology $G=(V, E, A)$ of order $n+$ $m(n, m>1)$ consists of two subgroups, $G_{1}=\left(V_{1}, E_{1}, A_{1}\right)$ and $G_{2}=\left(V_{2}, E_{2}, A_{2}\right)$, where $V_{1}=\left\{v_{1}, v_{2}, \ldots, v_{n}\right\}$ and $V_{2}=$ $\left\{v_{n+1}, v_{n+2}, \ldots, v_{n+m}\right\}$. Then, the set of finite index is defined by $L_{1}=\{1,2, \ldots, n\}$ and $L_{2}=\{n+1, n+2, \ldots, n+m\}$. In these two subgroups, $N_{1 i}=\left\{v_{j} \in V_{1}:\left(v_{i}, v_{j}\right) \in E\right\}$ and $N_{2 i}=\left\{v_{j} \in V_{2}:\left(v_{i}, v_{j}\right) \in E\right\}$ represent the neighbor set of node $v_{i}$, respectively. Then, we know that $V=V_{1} \cup V_{2}$ and $N_{i}=N_{1 i} \cup N_{2 i}$. For convenience, in this paper we only consider the case of two-group consensus problem.

Definition 3. Protocol (1) is said to reach a couple-group consensus asymptotically if the states of agents satisfy

(i) $\lim _{t \rightarrow \infty}\left\|x_{i}(t)-x_{j}(t)\right\|=0, \forall i, j \in L_{1}$;

(ii) $\lim _{t \rightarrow \infty}\left\|x_{i}(t)-x_{j}(t)\right\|=0, \forall i, j \in L_{2}$.

Lemma 4 (see [29]). For a connected bipartite graph $G$, the rank of $D+A$ is $n-1$, where $D$ and $A$ are the degree matrix and the adjacency matrix, respectively.

Lemma 5 (see [34]). If the graph $G$ contains a globally reachable node, 0 is the simple eigenvalue of its Laplacian matrix.

Lemma 6 (see [35]). For $\forall \gamma \in[0,1)$, if $\omega \in \mathbb{R}$, convex hull $\gamma \operatorname{Co}\left(0 \cup\left\{E_{i}(j \omega), i \in \mathbb{N}\right\}\right)$ will not enclose the point $(-1, j 0)$, where $E_{i}(j \omega)=\pi / 2 T \times e^{-j \omega T} / j \omega$ and $T$ denotes time delay.

Lemma 7 (see [36]). For $\omega \in \mathbb{R}$, convex hull $\gamma \mathrm{Co}(0 \cup$ $\left.\left\{E_{i}(j \omega), i \in \mathbb{N}\right\}\right)$ contains the set of $\bigcup_{i \in \mathbb{N}} G_{i}$.

\section{Couple-Group Consensus of Multiagent Networks with Multiple Delays}

In this section, we illustrate group consensus of multiagent networks with multiple time delays.

3.1. Couple-Group Consensus of Delayed Multiagent Networks with Bipartite Topologies. In [29], group consensus problems 
of the multiagent networks with protocol (2) and (3) were discussed and some sufficient conditions which can guarantee the achievement of group consensus were proposed as well:

$$
\begin{aligned}
& u_{i}(t)=-\sum_{v_{j} \in N_{i}} a_{i j}\left(x_{j}(t)+x_{i}(t)\right), \quad i \in \mathbb{N}, \\
& u_{i}(t)=-\sum_{v_{j} \in N_{i}} a_{i j}\left(x_{j}(t-\tau)+x_{i}(t-\tau)\right), \quad i \in \mathbb{N},
\end{aligned}
$$

where $\tau$ denotes the time delay.

Motivated by the related research works, we will discuss the group consensus problem of multiagent networks with different time delays. Consider the following protocol listed as

$$
u_{i}(t)=-\sum_{v_{j} \in N_{i}} a_{i j}\left(x_{j}\left(t-T_{i j}\right)+x_{i}\left(t-T_{i}\right)\right), \quad i \in \mathbb{N},
$$

where $T_{i j}$ denotes the communication delay between $v_{i}$ and $v_{j}$ and $T_{i}$ denotes the input delay of $v_{i}$. With (4), the closed-loop form of the networks (1) is

$$
\dot{x}_{i}(t)=-\sum_{v_{j} \in N_{i}} a_{i j}\left(x_{j}\left(t-T_{i j}\right)+x_{i}\left(t-T_{i}\right)\right), \quad i \in \mathbb{N} .
$$

Theorem 8. $\forall i \in \mathbb{N}$, if $\sum_{v_{j} \in N_{i}} a_{i j} T_{i}<\pi / 4$ can be satisfied, system (5) with bipartite topology will achieve couple-group consensus asymptotically.

Proof. Taking the Laplace transform to (5), we can obtain the characteristic equation as $\operatorname{det}\left(s I+D e^{-s T_{i j}}+A e^{-s T_{i}}\right)=0$, where $I$ denotes unit matrix, $D$ denotes the degree matrix, and $A$ denotes adjacency matrix. Define $F(s)=\operatorname{det}\left(s I+D e^{-s T_{i j}}+\right.$ $\left.A e^{-s T_{i}}\right)$, by the general Nyquist stability criterion, it is easy to know that the group consensus of the networks (5) can be achieved if and only if all zeros of $F(s)$ have negative real parts or $F(s)$ has a simple zero at $s=0$. Therefore, the following two cases are considered, respectively:

(i) When $s=0, F(s)=\operatorname{det}(D+A)$, by Lemma 4, we know that $F(s)$ contains a single zero at $s=0$.

(ii) When $s \neq 0$, define $P(s)=F(s) / s, G(s)=\left(D e^{-s T_{i j}}+\right.$ $\left.A e^{-s T_{i}}\right) / s$, then $P(s)=\operatorname{det}(I+G(s))$. It is easy to check that the discussion about the zeros of $F(s)$ is equal to the zeros of $P(s)$. So if all zeros of $P(s)$ have negative real parts, networks (5) will reach group consensus.

Define $s=j \omega$; by the general Nyquist stability criterion, it follows that all zeros of $F(s)$ have negative real parts for $\forall \omega \epsilon$ $\mathbb{R}$, if the Nyquist curve of the eigenvalue of $G(j \omega)$ does not enclose the point $(-1, j 0)$. By the Gerschgorin disk theorem, the equivalent of $G(j \omega)$ satisfies

$$
\lambda(G(j \omega)) \in \bigcup_{i \in \mathbb{N}} G_{i}
$$

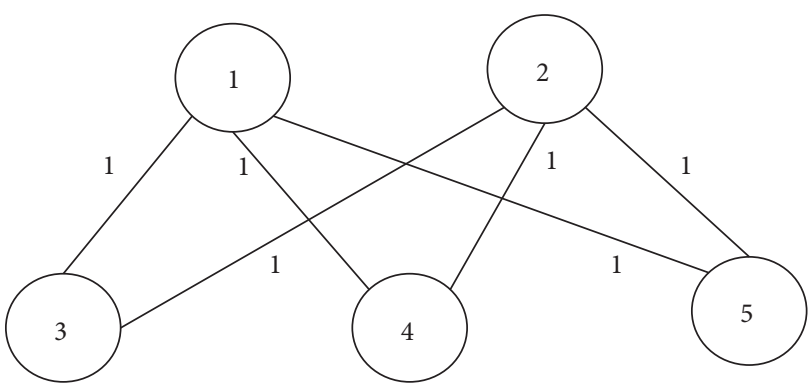

FIGURE 1: Topology of the multiagent networks (5).

$$
\begin{aligned}
& G_{i} \\
& =\left\{\zeta: \zeta \in C,\left|\zeta-\sum_{v_{j} \in N_{i}} a_{i j} \frac{e^{-j \omega T_{i}}}{j \omega}\right| \leq\left|\sum_{v_{j} \in N_{i}} a_{i j} \frac{e^{-j \omega T_{i}}}{j \omega}\right|\right\} .
\end{aligned}
$$

From (7), we know that the center of $G_{i}$ is $G_{i 0}(j \omega)=$ $\sum_{v_{j} \in N_{i}} a_{i j}\left(e^{-j \omega T_{i}} / j \omega\right)$. Suppose the intersection point $W_{i}$ connects the center of the disk and the origin point of complex plane. The track of point $W_{i}$ is $W_{i}(j \omega)=$ $2 \sum_{v_{j} \in N_{i}} a_{i j}\left(e^{-j \omega T_{i}} / j \omega\right)$. Based on Lemma 6 , as $W_{i}(j \omega)=$ $\gamma_{i} \times E_{i}(j \omega)$ and $\gamma_{i}<1$, we know that $\sum_{v_{j} \in N_{i}} a_{i j} T_{i}<\pi / 4$ holds. When $\gamma=\max \left\{\gamma_{i}, i \in \mathbb{N}\right\}$ and $\gamma<1$, for $\forall i \in$ $\mathbb{N}$, the next equation holds $\gamma \operatorname{Co}\left(0 \cup\left\{E_{i}(j \omega)\right\}\right) \supseteq \gamma_{i}(0 \cup$ $\left.\left\{E_{i}(j \omega)\right\}\right)=\operatorname{Co}\left(0 \cup\left\{W_{i}(j \omega)\right\}\right)$. By Lemma 6, noting that $(-1, j 0) \notin \gamma \operatorname{Co}\left(0 \cup\left\{E_{i}(j \omega), i \in \mathbb{N}\right\}\right)$ and based on Lemma 7 , as $\operatorname{Co}\left(0 \cup\left\{W_{i}(j \omega), i \in \mathbb{N}\right\}\right) \supseteq \bigcup_{i \in \mathbb{N}} G_{i}$, then $(-1, j 0) \notin \bigcup_{i \in \mathbb{N}} G_{i}$ holds. Thus, $\lambda(G(j w))$ does not enclose the point $(-1, j 0)$. That is to say, all zeros of $P(s)$ have negative real parts.

The proof of Theorem 8 is completed.

Remark 9. When $T_{i}=T_{i j}=\tau$, that is, all agents have same communication delays and input delays, it is obvious that protocols (3) and (4) are the same. That is to say, protocol (3) is a special form of (4). In [29-31], the cases where the input time delay is identical are discussed respectively. Whereas the time delays of the system including input time delay and communication time delays exist objectively and are usually different from each other, protocol (4) is more general.

Remark 10. Compared with the result in Theorem 8, it is known that the upper bound of time delays derived in [29] is relatively too broad. Meanwhile, according to the results in Theorem 8, it is shown that the group convergence of the networks is related to the input delays and adjacent weights among agents and independent of communication delays.

3.2. Couple-Group Consensus of First-Order Delayed Networks with the Topology Containing a Globally Reachable Node. In [24-27], based on the in-degree balance assumptions (A1) and control algorithm (8), the average-group consensus of multiagent networks with undirected, strongly connected, and balanced topology is explored, respectively. 
(A1): $\sum_{j=n+1}^{n+m} a_{i j}=0, \forall i \in L_{1}$, and $\sum_{j=1}^{n} a_{i j}=0, \forall i \in L_{2}$ :

$u_{i}(t)$

$$
= \begin{cases}\sum_{V_{j} \in N_{1 i}} a_{i j}\left(x_{j}(t)-x_{i}(t)\right)+\sum_{V_{j} \in N_{2 i}} a_{i j} x_{j}(t), \quad \forall i \in L_{1} \\ \sum_{V_{j} \in N_{2 i}} a_{i j}\left(x_{j}(t)-x_{i}(t)\right)+\sum_{V_{j} \in N_{1 i}} a_{i j} x_{j}(t), \quad \forall i \in L_{2} .\end{cases}
$$

Based on (8), Ji et al. [31] addressed multiagent networks (9) with undirected topology and delays:

$$
u_{i}(t)= \begin{cases}\sum_{V_{j} \in N_{1 i}} a_{i j}\left(x_{j}(t-\tau)-x_{i}(t-\tau)\right)+\sum_{V_{j} \in N_{2 i}} a_{i j} x_{j}(t-\tau), & \forall i \in L_{1} \\ \sum_{V_{j} \in N_{2 i}} a_{i j}\left(x_{j}(t-\tau)-x_{i}(t-\tau)\right)+\sum_{V_{j} \in N_{1 i}} a_{i j} x_{j}(t-\tau), & \forall i \in L_{2},\end{cases}
$$

where $\tau$ denotes time delay.

$$
u_{i}(t)= \begin{cases}\sum_{V_{j} \in N_{1 i}} a_{i j}\left(x_{j}\left(t-T_{i j}\right)-x_{i}\left(t-T_{i}\right)\right)+\sum_{V_{j} \in N_{2 i}} a_{i j} x_{j}\left(t-T_{i j}\right), & \forall i \in L_{1} \\ \sum_{V_{j} \in N_{2 i}} a_{i j}\left(x_{j}\left(t-T_{i j}\right)-x_{i}\left(t-T_{i}\right)\right)+\sum_{V_{j} \in N_{1 i}} a_{i j} x_{j}\left(t-T_{i j}\right) . & \forall i \in L_{2},\end{cases}
$$

With protocol (10), the closed-loop form of the networks

(1) is

$$
\dot{x}_{i}(t)= \begin{cases}\sum_{V_{j} \in N_{1 i}} a_{i j}\left(x_{j}\left(t-T_{i j}\right)-x_{i}\left(t-T_{i}\right)\right)+\sum_{V_{j} \in N_{2 i}} a_{i j} x_{j}\left(t-T_{i j}\right), & \forall i \in L_{1} \\ \sum_{V_{j} \in N_{2 i}} a_{i j}\left(x_{j}\left(t-T_{i j}\right)-x_{i}\left(t-T_{i}\right)\right)+\sum_{V_{j} \in N_{1 i}} a_{i j} x_{j}\left(t-T_{i j}\right) . & \forall i \in L_{2} .\end{cases}
$$

Theorem 11. Based on the in-degree balance conditions (A1), system (11) with the topology containing a globally reachable node can achieve couple-group consensus asymptotically if $\sum_{k=1, k \neq i}^{m+n} a_{i k} T_{i}<\pi / 4, i=1,2, \ldots, n+m$, holds.

Proof. Taking the Laplace transform to (11), its characteristic equation can be easily obtained as $\operatorname{det}(s I+L(s))=0$, where

$$
L(s)=\left(l_{i j}(s)\right)= \begin{cases}-a_{i j} e^{-s T_{i j}}, & j \neq i \\ \sum_{k=1, k \neq i}^{m+n} a_{i k} e^{-s T_{i}}, & j=i .\end{cases}
$$

The remainder of the proof process is similar to the proof of Theorem 8 , but here we omit it due to the space limitation.
Remark 12. When $T_{i}=T_{i j}=\tau$, protocols (9) and (10) are identical. Therefore, the related works in [24-27] can be seen as the special cases of Theorem 11 .

Remark 13. From the results in Theorems 8 and 11, it is shown that the achievement of the group consensus of the systems is determined by the input time delays and the coupling weight among the agents. Meanwhile, the node cannot tolerate bigger input delays if it owns a bigger coupling weight.

Corollary 14. When $T_{i}=\tau$, system (11) with the topology containing a globally reachable node can achieve couple-group consensus asymptotically if assumption (A1) is satisfied and $\tau<$ $\pi / 4 d$ holds, where $d=\max \left\{\sum_{k=1, k \neq i}^{m+n} a_{i k}\right\}, i=1,2, \ldots, m+n$.

Remark 15. The result in Corollary 14 is a special case of Theorem 11, and it is consistent with the relevant conclusions 


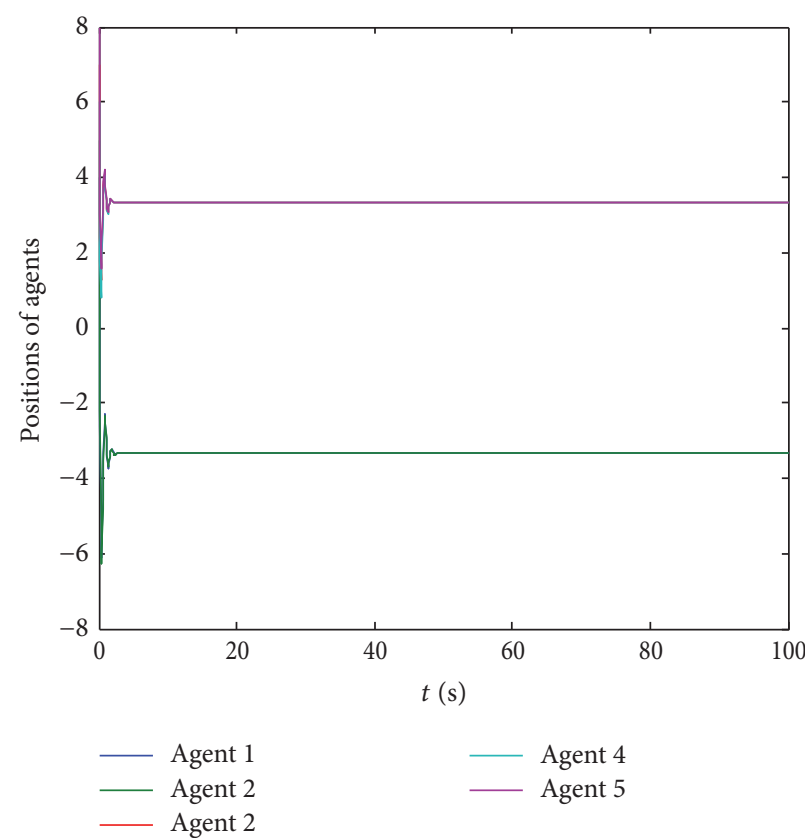

(a) $T_{i j}=0.1 \mathrm{~s}$

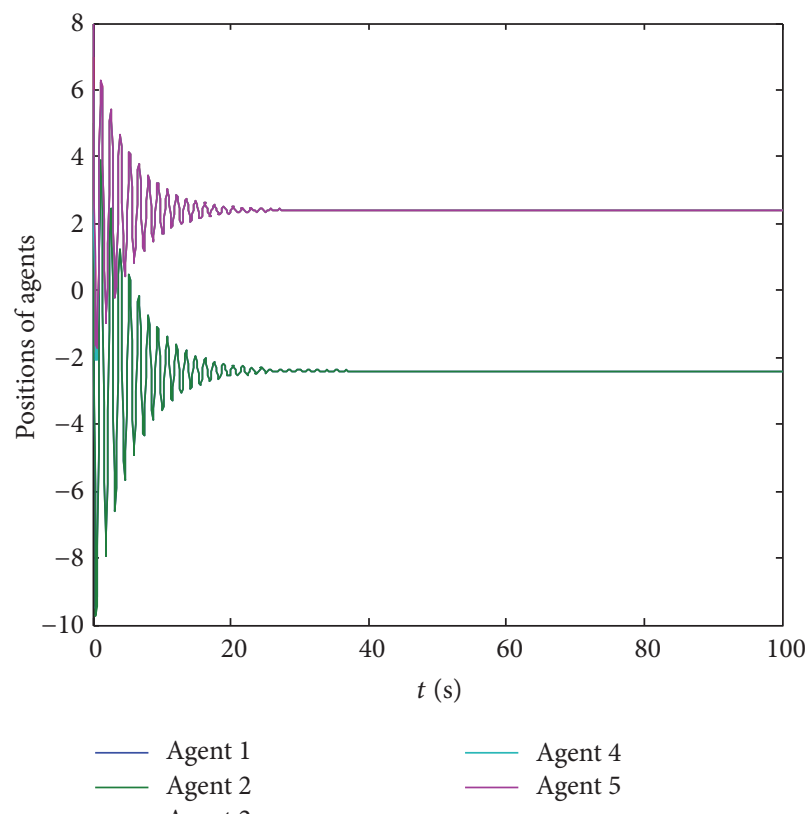

(c) $T_{i j}=0.3 \mathrm{~s}$

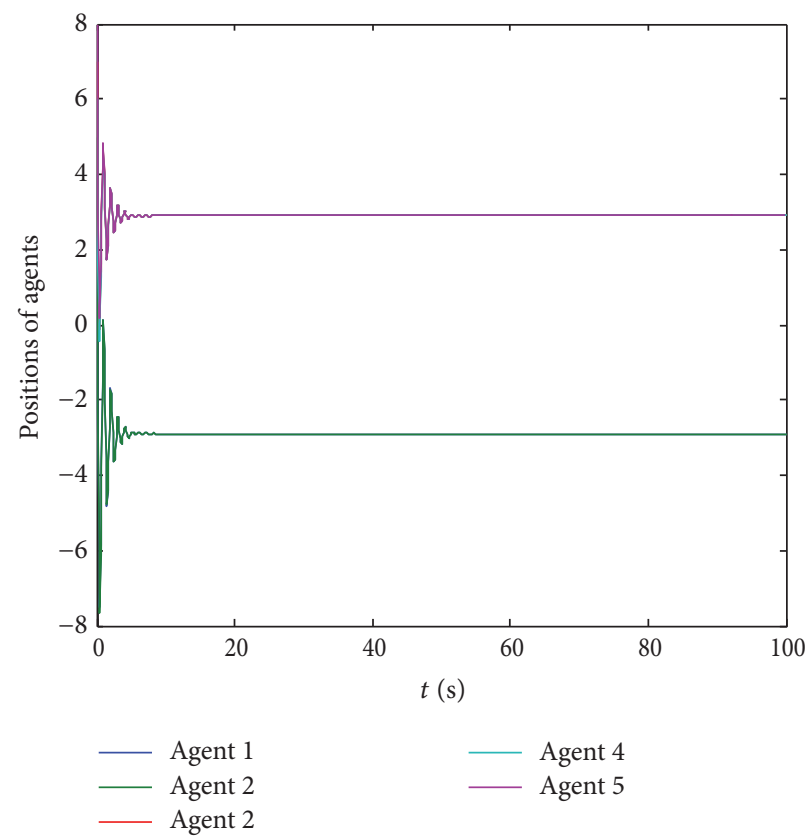

(b) $T_{i j}=0.2 \mathrm{~s}$

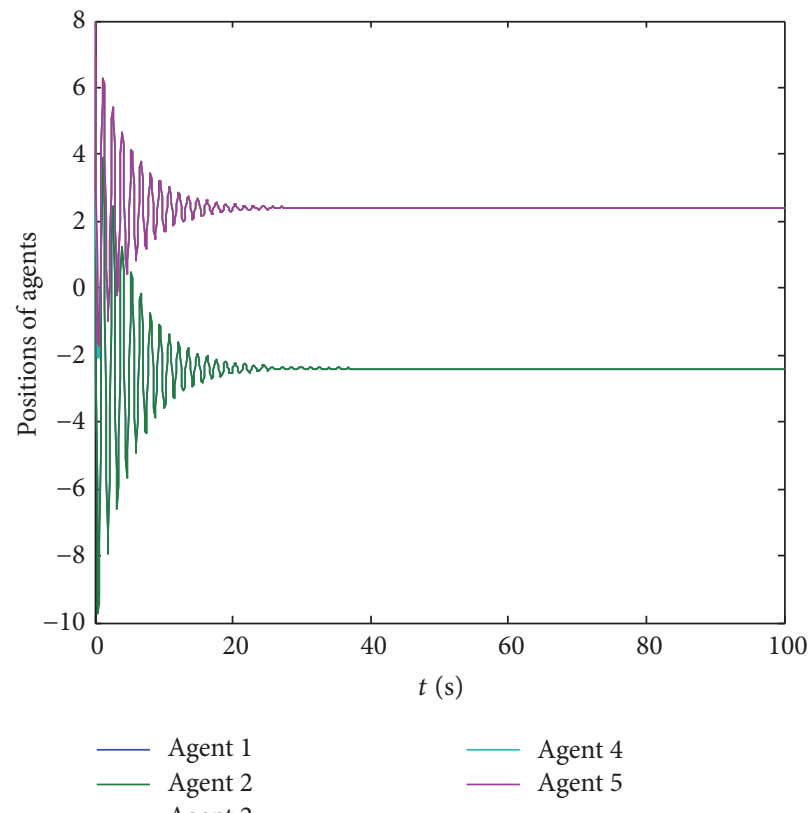

(d) $T_{i j}=0.4 \mathrm{~s}$

FIGURE 2: State trajectories of the agents in the networks (5) with delays.

in [31]. Meanwhile, it is shown that the tolerant upper bound of input time delay in the system is determined by the node who owns the max coupling weight.

\section{Simulation Examples}

In this section, some simulation examples will be given to show the effectiveness and correctness of our findings.
Experiment 1. Suppose the topology of the networks (5) is shown as Figure 1. In Figure 1, nodes $v_{1}, v_{2}, v_{3}, v_{4}$, and $v_{5}$ belong to the two different subgroups, respectively. In the experiment, initial states of agents are generated from 0 to 10 randomly and the input delays of agents are set as follows: $T_{1}=0.2 \mathrm{~s}, T_{2}=0.2 \mathrm{~s}$, and $T_{3}=T_{4}=T_{5}=0.1 \mathrm{~s}$. It is easy to verify that all input delays satisfy the conditions in Theorem 8 . Meanwhile, for simplicity, we only consider the case that the communication time delays among the agents are same and 


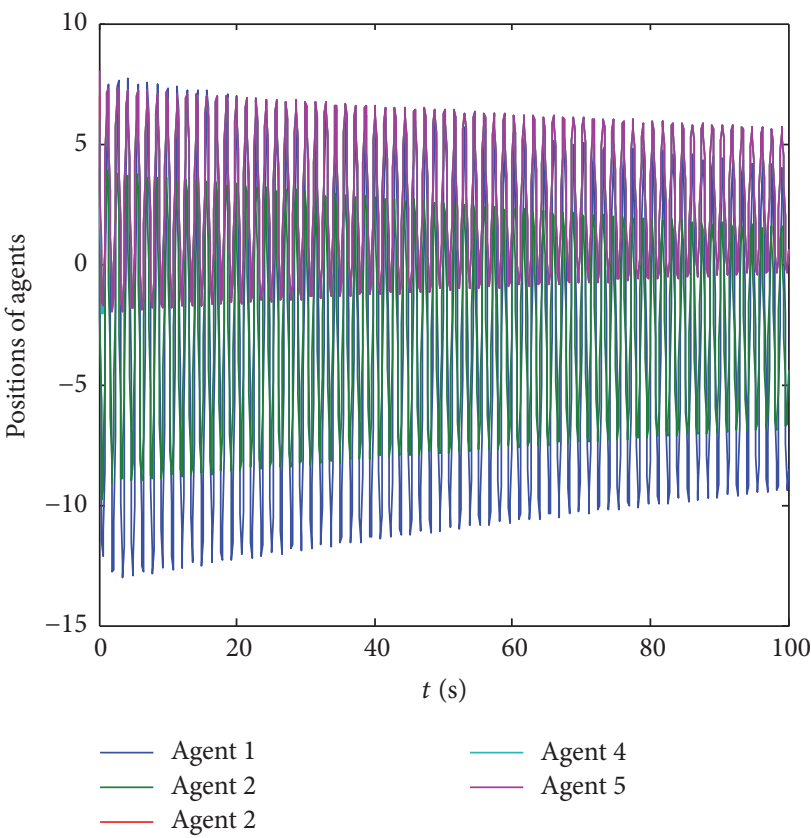

(a) $T_{1}=0.26 \mathrm{~s}$

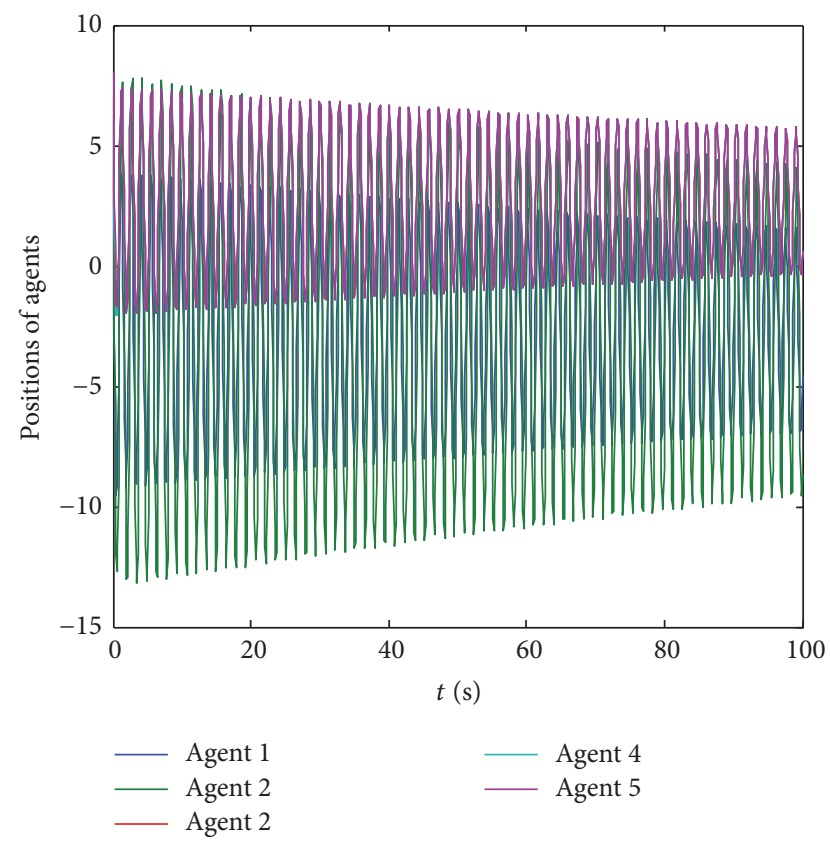

(b) $T_{2}=0.26 \mathrm{~s}$

FIGURE 3: State trajectories of the agents in the networks (5) with diverse input delays when $T_{i j}=0.1 \mathrm{~s}$.

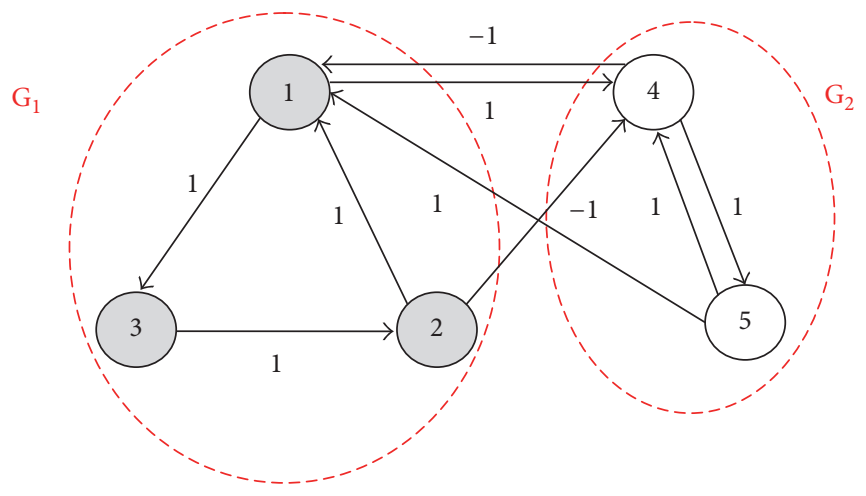

FIGURE 4: The topology of the multiagent networks (11).

set it as $T_{i j}=0.1,0.2,0.3,0.4 \mathrm{~s}, i, j=1,2,3,4,5$, respectively. The trajectories of the agents are shown in Figure 2 for these four cases. According to the results, it is shown that networks (5) can achieve couple-group consensus asymptotically, respectively. At the same time, by comparing the trajectories with different communication delays, it is clear that communication time delays will not affect convergence properties of the networks but can affect the convergence rate of the system. Furthermore, with the decrease of the communication delays, the faster systems will convergence.

From Figure 1, by Theorem 8, the allowed input delays of $v_{1}$ and $v_{2}$ should satisfy $T_{i}<\pi / 12=0.26 \mathrm{~s}$, which means that group consensus of the networks can be achieved. Next, the following two cases are considered and the state trajectories of the networks (5) are shown in Figure 3: (i) Reset $T_{1}=0.26 \mathrm{~s}$; input delays of other nodes are not changed.

(ii) Reset $T_{2}=0.26 \mathrm{~s}$; input delays of other nodes are not changed.

From the results in Figure 3, it is illustrated that networks (5) will not reach couple-group group consensus in these three cases. Therefore, the results of simulation experiments further verity the correctness and effectiveness of the algebraic criteria in Theorem 8 .

From Figure 1, it is easy to get its eigenvalues are $0,-2.0,-2.0,-3.0,-5.0$, respectively. Based on the bound of time delays $0<\tau<\left(-\pi / 2 \lambda_{m}\right)$ s presented in [29], the bound of time delays presented in [29] has $\tau \in(0,0.314) \mathrm{s}$. Compared with the results in Figure 3, the upper bound of the time delay is too broader. 


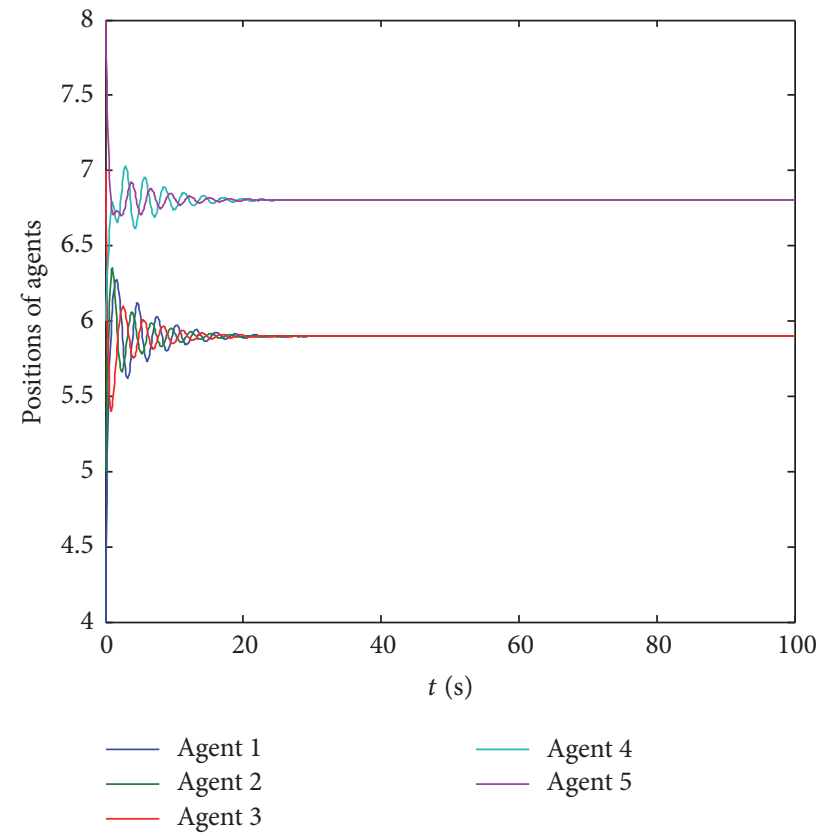

(a) $T_{1}=0.1 \mathrm{~s}$

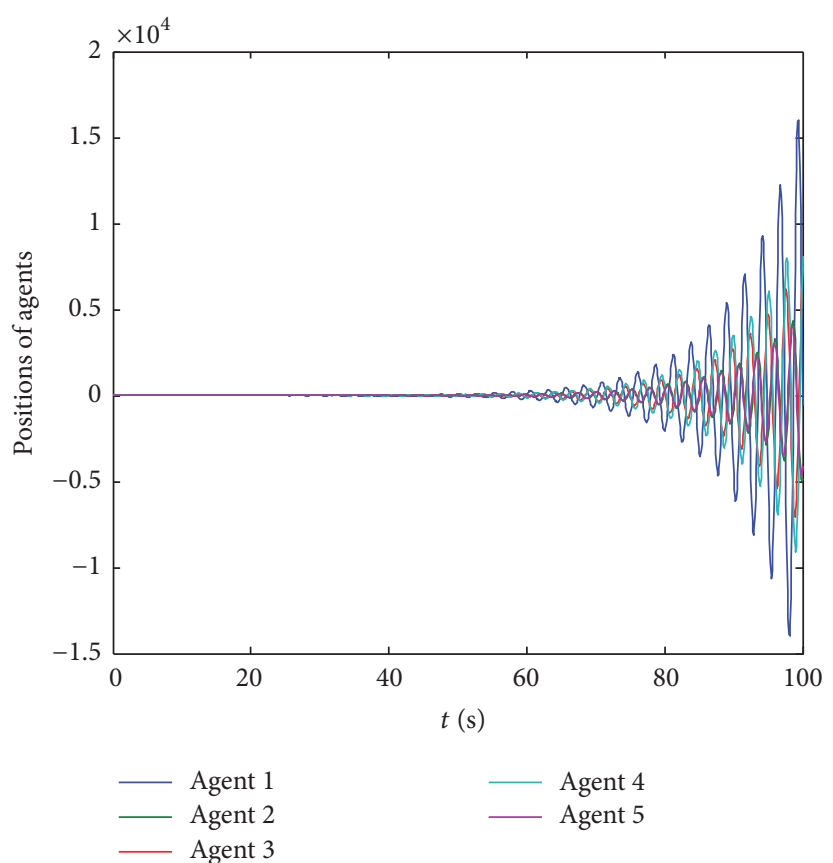

(b) $T_{1}=0.4 \mathrm{~s}$

FIGURE 5: State trajectories of the agents in the networks (11).

Experiment 2. Given the dynamical networks (11) with 5 nodes, the topology and connection weights between nodes are shown in Figure 4. Agents $v_{1}, v_{2}$, and $v_{3}$ are in a group while agents $v_{4}$ and $v_{5}$ are in another group. Meanwhile, the topology of multiagent networks contains a globally reachable node and satisfies the in-degree balance assumption (A1). Similarly, the initial states of agents are generated from 0 to 10 randomly, and communication delay of all agents is set as $T_{i j}=0.3 \mathrm{~s}$; input delays of the agents are $T_{1}=0.1 \mathrm{~s}$, $T_{2}=0.7 \mathrm{~s}, T_{3}=0.2 \mathrm{~s}, T_{4}=0.5 \mathrm{~s}$, and $T_{5}=0.2 \mathrm{~s}$. It is easy to verify the conditions in Theorem $11 \mathrm{can}$ be satisfied. The state trajectories of the agents in networks (11) are shown in Figure 5(a); it is clear that the networks can achieve couplegroup consensus.

Next, the upper bound of time delay presented in Theorem 11 will be checked. To node $v_{1}$, it has $\widetilde{d}_{1}=2$ according the topology shown in Figure 4. By Theorem 11, the input delay of node $v_{1}$ should satisfy $T_{1}<\pi / 8 \mathrm{~s}$. If set $T_{1}=0.4 \mathrm{~s}$, obviously, the condition in Theorem 11 is not satisfied. In the case of communication and input delays of other nodes keeping unchanged, described in Figure 5(b), couple-group consensus will not be achieved.

Experiment 3. Based on the Experiment 2, the validity of Corollary 14 will be verified here. From Corollary 14, we can get $\tau<\pi / 8$ s. So the following two cases are considered: $\tau=0.35 \mathrm{~s}$ and $\tau=0.4 \mathrm{~s}$. The trajectories of the agents are shown in Figure 6, respectively. The results illustrate that the couple-group consensus of the system can be achieved if the conditions in Corollary 14 are satisfied.

\section{Conclusion}

This paper investigated the couple-group consensus problem of multiagent networks with time delays. By applying the theory of frequency-domain, some criteria are derived which can guarantee the realization of group consensus. Meanwhile, the upper bounds of input time delays that the systems can be tolerant are proposed analytically as well. From the results, it is shown that both the input delays and the coupling weights between the agents play an important part in the achievement of group consensus. However, communication delays just only affect the convergence rate of networks. Thus, convergence performance of the system can be improved by reducing the communication time delays.

\section{Competing Interests}

All authors declare they have no competing interests.

\section{Acknowledgments}

This work was supported by the Natural Science Foundation Project of Chongqing Science and Technology Commission under Grant no. cstc2014jcyjA40047 and in part by the Scientific and Technological Research Program of Chongqing Municipal Education Commission under Grant no. KJ1400403 and in part awarded by State Scholarship Fund of China Scholarship Council. 


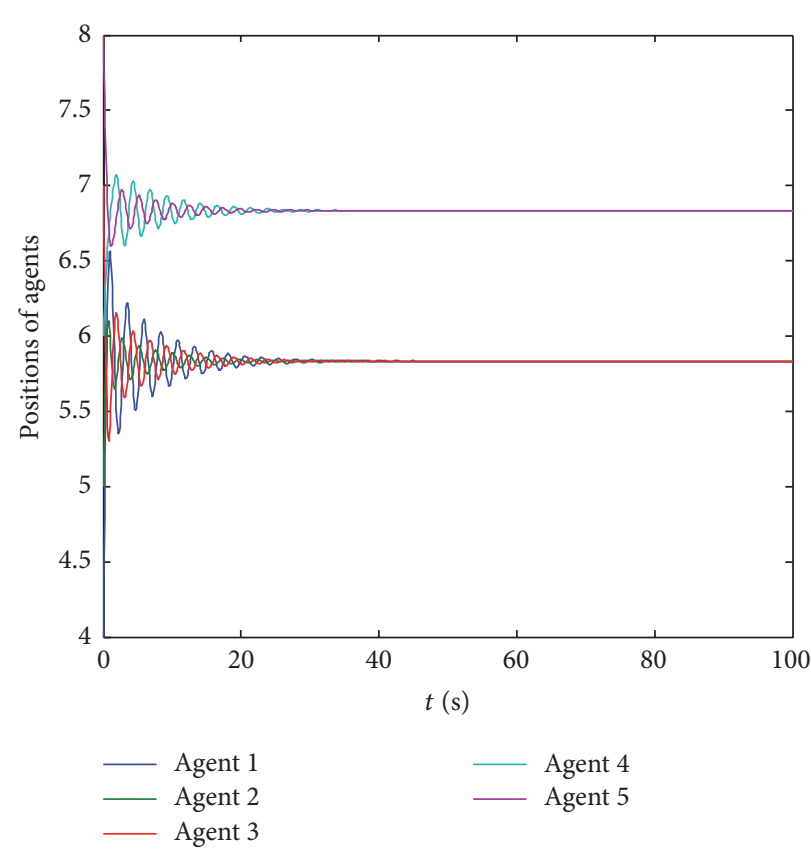

(a) $\tau=0.35 \mathrm{~s}$

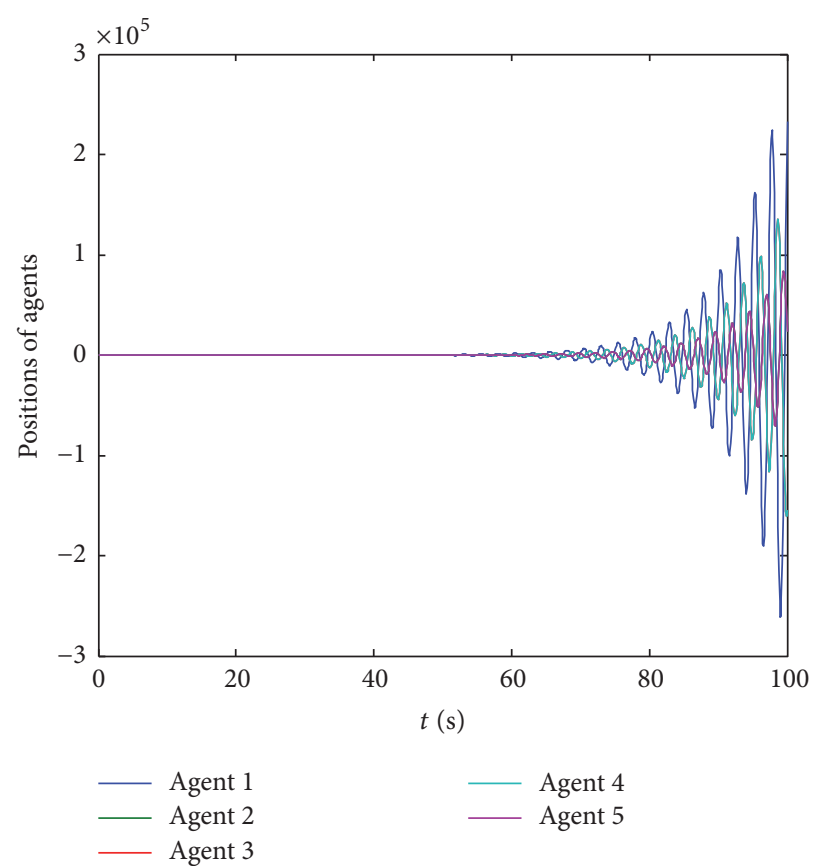

(b) $\tau=0.4 \mathrm{~s}$

Figure 6: State trajectories of the agents in the networks (11) when $T_{i}=\tau$.

\section{References}

[1] H. Shi, L. Wang, and T. Chu, "Swarming behavior of multi-agent systems," Journal of Control Theory and Applications, vol. 2, no. 4, pp. 313-318, 2004.

[2] A. Ghaffari, "Congestion control mechanisms in wireless sensor networks: a survey," Journal of Network and Computer Applications, vol. 52, pp. 101-115, 2015.

[3] C. Sergiou, V. Vassiliou, and A. Paphitis, "Hierarchical Tree Alternative Path (HTAP) algorithm for congestion control in wireless sensor networks," Ad Hoc Networks, vol. 11, no. 1, pp. 257-272, 2013.

[4] Z. C. Li and X. P. Xue, "Cucker-Smale flocking under rooted leadership with free-will agents," Physica A. Statistical Mechanics and Its Applications, vol. 410, pp. 205-217, 2014.

[5] S. Martin, "Multi-agent flocking under topological interactions," Systems \& Control Letters, vol. 69, no. 1, pp. 53-61, 2014.

[6] W. W. Yu, G. R. Chen, and M. Cao, "Some necessary and sufficient conditions for second-order consensus in multi-agent dynamical systems," Automatica, vol. 46, no. 6, pp. 1089-1095, 2010.

[7] X. J. Wu and H. T. Lu, "Outer synchronization of uncertain general complex delayed networks with adaptive coupling," Neurocomputing, vol. 82, pp. 157-166, 2012.

[8] Z. J. Tang, T. Z. Huang, J. L. Shao, and J. P. Hu, "Consensus of second-order multi-agent systems with nonuniform timevarying delays," Neurocomputing, vol. 115, pp. 169-177, 2012.

[9] B. Liu, X. L. Wang, H. S. Su, Y. P. Gao, and L. Wang, "Adaptive second-order consensus of multi-agent systems with heterogeneous nonlinear dynamics and time-varying delays," Neurocomputing, vol. 118, pp. 289-300, 2013.

[10] H. Yan, Y. Shen, H. Zhang, and H. Shi, "Decentralized eventtriggered consensus control for second-order multi-agent systems," Neurocomputing, vol. 133, pp. 18-24, 2014.
[11] T. C. Zhang and H. Yu, "Average consensus for directed networks of multi-agent with time-varying delay," Computer Science, vol. 6145, pp. 723-730, 2010.

[12] D. M. Xie and L. Teng, "Seconde-order group consensus for multi-agent systems with time delays," Neurocomputing, vol.153, pp. 133-139, 2015.

[13] Y. Z. Wang, C. H. Zhang, and Z. B. Liu, "A matrix approach to graph maximum stable set and coloring problems with application to multi-agent systems," Automatica, vol. 48, no. 7, pp. 1227-1236, 2012.

[14] J.-W. Yi, Y.-W. Wang, and J.-W. Xiao, "Reaching cluster consensus in multi-agent systems," in Proceedings of the 2nd International Conference on Intelligent Control and Information Processing (ICICIP '11), pp. 569-573, Harbin, China, July 2011.

[15] C. Tan, G. P. Liu, and G. R. Duan, "Group consensus of networked multi-agent systems with directed topology," IFAC Proceedings Volumes, vol. 44, no. 1, pp. 8878-8893, 2011.

[16] H.-X. Hu, L. Yu, W.-A. Zhang, and H. Song, "Group consensus in multi-agent systems with hybrid protocol," Journal of the Franklin Institute, vol. 350, no. 3, pp. 575-597, 2013.

[17] H. Y. Zhao, J. H. Park, and Y. L. Zhang, "Couple-group consensus for second-order multi-agent systems with fixed and stochastic switching topologies," Applied Mathematics and Computation, vol. 232, pp. 595-605, 2014.

[18] H. W. Ma, D. R. Liu, D. Wang, F. X. Tan, and C. Li, "Centralized and decentralized event-triggered control for group consensus with fixed topology in continuous time," Neurocomputing, vol. 161, pp. 267-276, 2015.

[19] X. Liu and T. Chen, "Cluster synchronization in directed networks via intermittent pinning control," IEEE Transactions on Neural Networks, vol. 22, no. 7, pp. 1009-1020, 2011. 
[20] H. Su, Z. Rong, M. Z. Q. Chen, X. Wang, G. Chen, and H. Wang, "Decentralized adaptive pinning control for cluster synchronization of complex dynamical networks," IEEE Transactions on Cybernetics, vol. 43, no. 1, pp. 394-399, 2013.

[21] G. Wen, W. Yu, J. Wang, D. Xu, and J. Cao, "Distributed nodeto-node consensus of multi-agent systems with time-varying pinning links,” Neurocomputing, vol. 149, pp. 1387-1395, 2015.

[22] X. F. Liao and L. H. Ji, "On pinning group consensus for dynamical multi-agent networks with general connected topology," Neurocomputing, vol. 135, pp. 262-267, 2014.

[23] L. Ji, Q. Liu, and X. Liao, "On reaching group consensus for linearly coupled multi-agent networks," Information Sciences, vol. 287, pp. 1-12, 2014.

[24] J. Y. Yu and L. Wang, "Group consensus of multi-agent systems with undirected communication graph," in Proceedings of the Asian Control Conference, Beijing, China, 2009.

[25] J. Yu and L. Wang, "Group consensus of multi-agent systems with directed information exchange," International Journal of Systems Science, vol. 43, no. 2, pp. 334-348, 2012.

[26] J. Y. Yu and L. Wang, "Output feedback stabilization of networked control systems via switched system approach," International Journal of Control, vol. 82, no. 9, pp. 1665-1677, 2009.

[27] J. Y. Yu and L. Wang, "Group consensus in multi-agent systems with switching topologies and communication delays," Systems \& Control Letters, vol. 59, no. 6, pp. 340-348, 2010.

[28] M. H. Wang and K. Uchida, "Cluster Consensus of Multiagent system with communication delay," in Proceedings of the International Conference on Control Automation and Systems, Gwangiu, Korea, October 2013.

[29] Q. Wang, Y. Z. Wang, and R. M. Yang, "The design and analysis of group-consensus protocol for a class of multi-agent systems," Control and Decision, vol. 28, no. 3, pp. 369-373, 2013.

[30] X. Y. Du, Y. Z. Wang, and Q. Wang, "Weighted group-consensus analysis of multi-agent systems with and without time-delay network," in Proceedings of the 34th Chinese Control Conference (CCC '15), pp. 6900-6905, Hangzhou, China, July 2015.

[31] L.-H. Ji, X.-F. Liao, and Q. Liu, "Group consensus analysis of multi-agent systems with delays," Acta Physica Sinica, vol. 61, no. 22, Article ID 220202, 2012.

[32] J. Lianghao and Z. Xinyue, "Group consensus of second-order multi-agent networks with multiple time delays," in Proceedings of the International Conference on Applied Mathematics, Simulation and Modelling (AMSM '16), pp. 437-441, Beijing, China, May 2016.

[33] C. Godsil and G. Royle, Algebraic Graph Theory, vol. 207 of Graduate Texts in Mathematics, Springer, New York, NY, USA, 2001.

[34] W. Ren and R. W. Beard, "Consensus seeking in multiagent systems under dynamically changing interaction topologies," IEEE Transactions on Automatic Control, vol. 50, no. 5, pp. 655$661,2005$.

[35] Y. Tian and H. Y. Yang, "Stability of distributed congestion control with diverse communication delays," Intelligent Control and Automation, vol. 2, pp. 1438-1442, 2004.

[36] H.-Y. Yang, S.-W. Tian, and S.-Y. Zhang, "Consensus of multiagent systems with heterogeneous delays and leader-following," Acta Electronica Sinica, vol. 39, no. 4, pp. 872-876, 2011. 


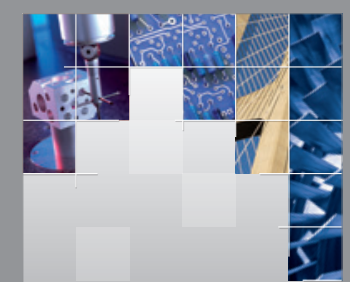

\section{Enfincering}
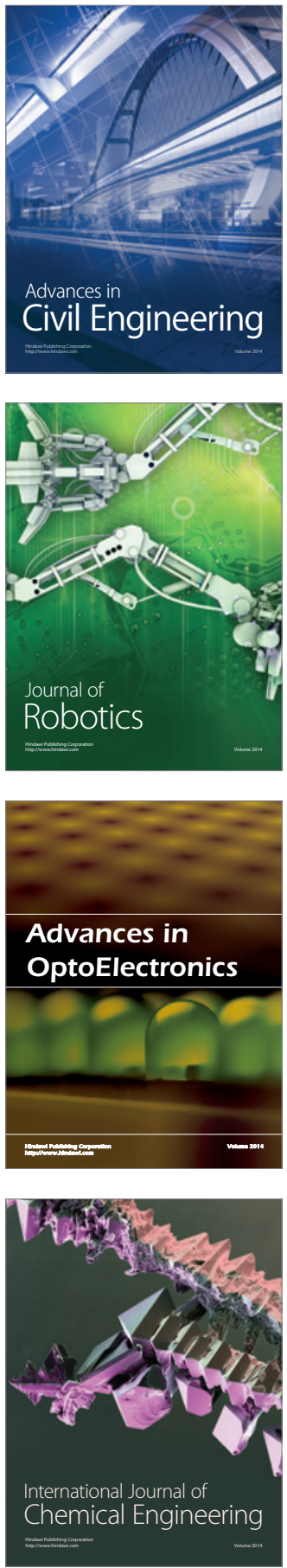

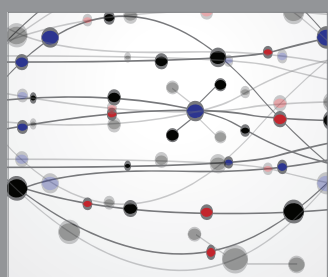

The Scientific World Journal

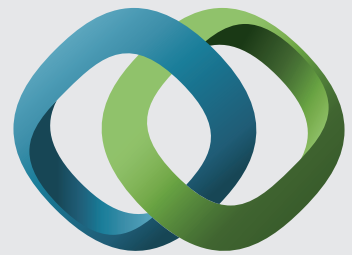

\section{Hindawi}

Submit your manuscripts at

http://www.hindawi.com
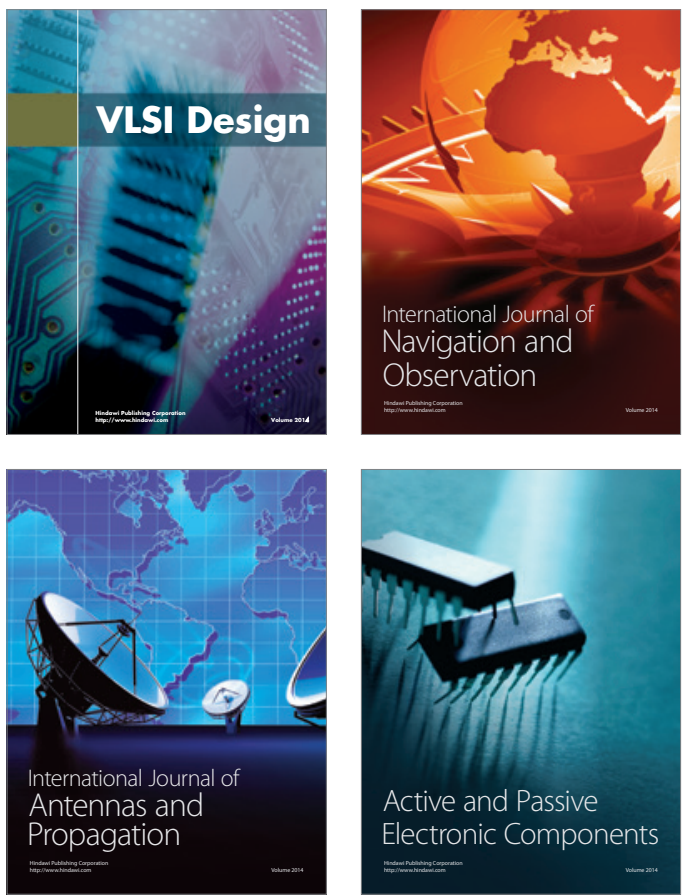
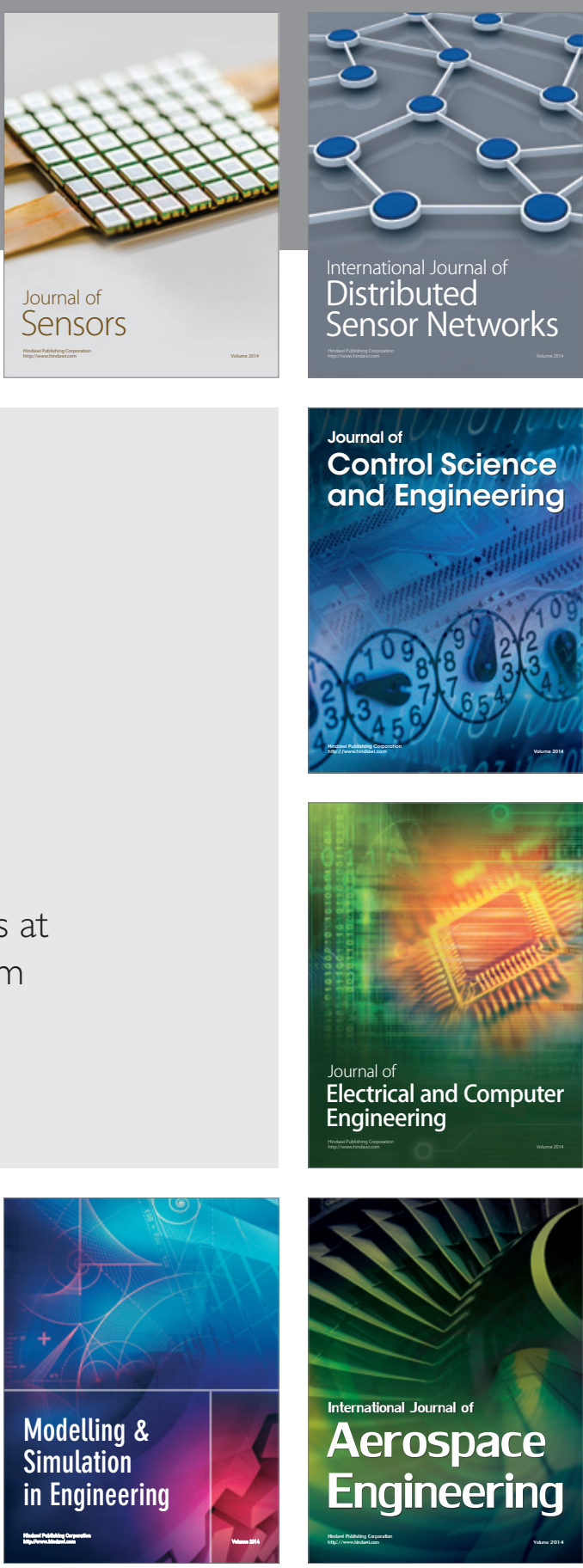

International Journal of

Distributed

Sensor Networks

Journal of

Control Science

and Engineering
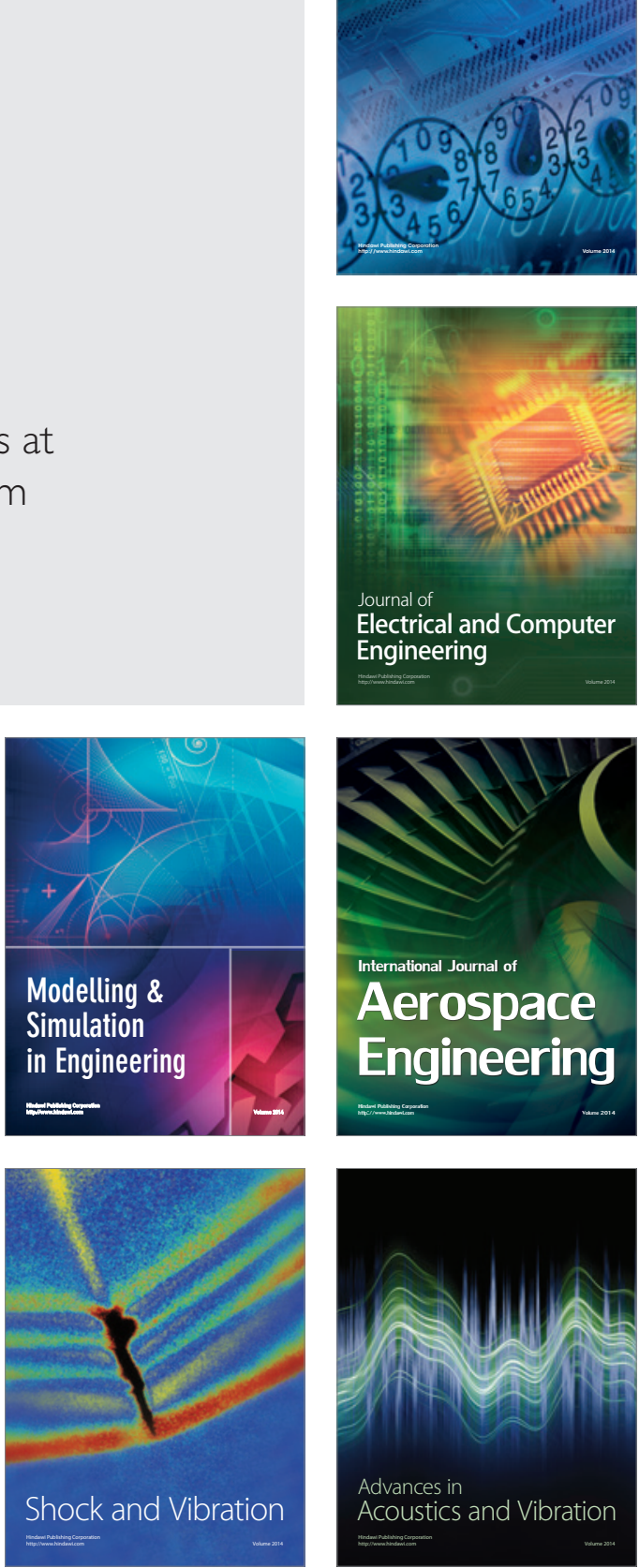